\title{
Foveal cone photoreceptor involvement in primary open-angle glaucoma
}

\author{
Martijn J. Kanis • Hans G. Lemij • \\ Tos T. J. M. Berendschot • Jan van de Kraats • \\ Dirk van Norren
}

Received: 1 September 2009 /Revised: 15 January 2010 / Accepted: 5 February 2010/Published online: 7 March 2010

(C) The Author(s) 2010. This article is published with open access at Springerlink.com

\begin{abstract}
Background To test whether foveal cone photoreceptors are impaired in primary open-angle glaucoma (POAG).

Methods Nineteen POAG eyes with central glaucomatous visual field defects, and 34 age-matched control eyes were included. Fundus reflectometry, together with a model fit procedure, provided information on a set of parameters: lens optical density, macular pigment optical density, melanin, blood, the directional cone reflectance (Rd), a measure for foveal cone photoreceptor integrity, and RILM, the reflectance at the inner limiting membrane. Optical coherence tomography (OCT) was performed to assess macular thickness. A Kolmogorov-Smirnov $Z$-test was used to compare parameters between the two groups.

Results Median age (range) was 55.1 (24.7-73.3) years in the control subjects, and $60.1(20.7-77.0)$ years in the POAG patients $(P=0.24)$. Of all eight model parameters, only Rd and RILM were significantly lower in POAG.
\end{abstract}

This work was supported by the Dr. F.P. Fischer Foundation, Utrecht, The Netherlands.

The authors have full control of all primary data, and agree to allow Graefe's Archive for Clinical and Experimental Ophthalmology to review their data upon request.

M. J. Kanis · J. van de Kraats · D. van Norren

Department of Ophthalmology,

University Medical Center Utrecht,

Utrecht, The Netherlands

H. G. Lemij

The Rotterdam Eye Hospital,

Rotterdam, The Netherlands

T. T. J. M. Berendschot $(\bowtie)$

University Eye Clinic Maastricht,

P.O. Box 5800, 6202 AZ Maastricht, The Netherlands

e-mail: t.berendschot@ohk.unimaas.nl
Median Rd (range) was $2.21(0.64-4.93) \%$ in the control subjects and $1.19(0.08-3.60) \%$ in the POAG patients $(P=$ $0.003)$. Median RILM (range) was $0.15(0.00-1.08) \%$ in the control subjects, and $0.08(0.01-0.29) \%$ in the POAG patients $(P<0.001)$. Rd showed no linear relationship with central retinal sensitivity on Visual Field test in POAG patients. Retinal thickness of the inner $1-3 \mathrm{~mm}$ ring and the outer 3-6 $\mathrm{mm}$ ring on OCT, centered on the fovea, was significantly lower in POAG patients than in control subjects. Conclusions The integrity of the foveal cone outer segments, and the reflectance of the central ILM were impaired in POAG with advanced central visual field defects.

Keywords Glaucoma · Cone · Photoreceptor · ILM ·

Foveal reflection analysis $\cdot$ OCT

\section{Introduction}

Glaucoma is a group of ocular disorders characterized by progressive loss of retinal ganglion cells and their axons, associated with visual function loss and, frequently, elevated intra-ocular pressure (IOP). It is the second leading cause of blindness in the world, affecting more than 66 million individuals. Primary open-angle glaucoma (POAG) is estimated to affect 33.2 million people worldwide, of which an estimated $10 \%$ have severe visual impairment [1].

Glaucoma primarily involves the most inner retinal layer. Whether the outer retinal layer can be involved in glaucoma, in particular the photoreceptors outside the parapapillary region, is still unclear. Panda and Jonas counted the nuclei of retinal photoreceptors (mainly rods) in histological slides of 23 enucleated eyes with painful angle-closure glaucoma secondary to penetrating corneal injuries [2]. The photoreceptor count was significantly 
lower in the glaucoma eyes than in the control group. The authors concluded that this suggested a more widespread glaucomatous involvement of ocular tissues, including changes of the deep retinal layers outside the area around the optic disc. To verify whether their finding also applied to POAG, Kendell et al. compared the estimated number of photoreceptors (mainly rods) in 14 human eyes with a history of POAG with nine age-matched, normal eyes [3]. They concluded that glaucomatous and normal eyes did not differ in their photoreceptor count. Neither did they find any association between photoreceptor count and the severity of glaucoma. Wygnanski et al. counted the number of cones and ganglion cells in monkeys with experimental glaucoma [4]. The authors found no marked parafoveal cone loss despite extensive ganglion cell damage. They concluded that the decreased photoreceptor count found by Panda and Jonas [2] was probably caused by the ocular trauma, rather than by the secondary glaucoma.

A study conducted by Nork et al. [5] showed swelling but no loss in the number of red- and green-sensitive cones in human eyes with presumed POAG, and in monkey eyes with experimental glaucoma. The authors concluded that the photoreceptors were affected by chronically elevated intraocular pressure. They proposed a mechanism in which the lowered choroidal blood flow in POAG causes photoreceptor ischemia, leading to a decreased reuptake of glutamate, the major excitatory neurotransmitter of the photoreceptors. Higher levels of extracellular glutamate might lead to overstimulation of bipolar cells and eventually of ganglion cells, resulting in apoptosis of the ganglion cells.

Electrophysiological findings indicating outer retinal involvement in POAG have also been reported [6-10], but they have not been confirmed in others [11-13].

In conclusion, there is little evidence that photoreceptors are affected in POAG. In our study, we tested whether foveal cone photoreceptors are impaired in POAG. To that end, we quantitatively compared the integrity of the foveal cones between healthy control subjects and POAG patients with central visual field defects within 8 degrees from the fovea, using a very sensitive reflectometric technique related to the optical Stiles-Crawford effect. Because, on the basis of the literature, we were unsure whether we would find any effect, we decided to start our investigation with severe cases of POAG.

\section{Materials and methods}

Participants

Healthy volunteers were recruited via an advertisement in a local newspaper. POAG patients were enrolled among those visiting the outpatient clinic of the ophthalmology depart- ment of the University Medical Center Utrecht (UMC Utrecht). After written informed consent was obtained, all participants (healthy control subjects and POAG patients) were questioned about their medical history. An autorefractometer (Auto Refkeratometer Speedy-K, Nikon Corporation, Tokyo, Japan) was used to measure any refractive error of both eyes in every participant. These data were used to obtain the best-corrected visual acuity (BCVA) of both eyes in all participants by means of an ETDRS chart at 4 meters. Next, a visual field examination of the study eye (see later) was performed in both the healthy control subjects and the POAG patients, by using the Octopus Perimeter 101 (Haag-Streit International, Köniz, Switzerland). In the healthy control group, the right eye was arbitrarily used as the study eye, and was examined with the 32 program (76 test points from $0-30^{\circ}$, equidistant grid with $6^{\circ}$ spacing). In the POAG group, the eye with the most advanced central visual field defects upon prior testing, together with a BCVA of maximally 0.7 LogMAR (equivalent to a Snellen visual acuity $\geq 20 / 100$ ) was selected, and was perimetrically examined with program $\mathrm{C} 08$ (56 test points from $0-8^{\circ}$, equidistant grid with $2^{\circ}$ spacing). After the visual field examination, tropicamide $0.5 \%$ and phenylephrine $5 \%$ eye drops were used for pupil dilation of the study eyes. We then aimed at performing five representative measurements with the foveal reflection analyzer (FRA, see next paragraph). In addition, the intraocular pressure (IOP) of the study eye was measured by means of Goldmann applanation tonometry in all participants. An OCT scan (Stratus OCT 3000, software version 4.0.1, Carl Zeiss Meditec Inc, Dublin, CA, USA) of the macula of the study eye was thereafter performed, in all 34 control subjects. Because the OCT scanner was not available at all times, an OCT scan was not performed in three POAG patients. Finally, $50^{\circ}$ images of the macular area of the study eye were taken (Zeiss FF-450 fundus camera) to detect any macular abnormalities. The inclusion and exclusion criteria are summarized in Table 1.

The age-matched control group consisted of 34 right eyes of 17 women and 17 men. In total, 170 measurements were performed and used in the control group. The POAG group consisted of 95 measurements from 11 right eyes and eight left eyes of nine women and ten men. Table 2 shows characteristics of all 19 patients.

This study was conducted in accordance with the tenets of the declaration of Helsinki, and approved by the local Medical Ethics Committee.

Measurement technique

The foveal reflection analyzer (FRA) was used to measure the cone-specific directional reflectance. A prototype FRA was detailed by Zagers et al. [14]; the present version was recently described by Van de Kraats and Van Norren [15]. 
Table 1 Inclusion and exclusion criteria for control subjects and POAG patients
* Glaucomatous VF defects were defined as three adjacent points in one quadrant with $p<0.05$.

$\dagger$ Exclusion if reliability

factor $>15 \%$

\begin{tabular}{|c|c|c|}
\hline & Control subjects & POAG patients \\
\hline \multirow[t]{5}{*}{ Inclusion criteria } & age $\geq 18$ years & age $\geq 18$ years \\
\hline & no visual complaints & diagnosed with $\mathrm{POAG}>2$ years \\
\hline & no history of ophthalmic disease & $\begin{array}{l}\text { central glaucomatous VF defects } \\
\text { within } 8 \text { degrees from fovea }\end{array}$ \\
\hline & $\begin{array}{l}\text { no history of ophthalmic surgery, } \\
\text { including IOL implantation }\end{array}$ & no diabetes mellitus \\
\hline & no diabetes mellitus & no history of other retinal abnormalities \\
\hline \multirow[t]{5}{*}{ Exclusion criteria } & $\begin{array}{l}\text { BCVA>0.1 LogMAR } \\
\quad(\text { Snellen } \mathrm{BCVA}<80 / 100)\end{array}$ & $\begin{array}{l}\text { BCVA >0.7 LogMAR } \\
\quad(\text { Snellen BCVA<20/100) }\end{array}$ \\
\hline & $\mathrm{IOP}>21 \mathrm{mmHg}$ & reliability VF test low ${ }^{\dagger}$ \\
\hline & presence of glaucomatous VF defects ${ }^{*}$ & $\begin{array}{l}\text { macular abnormalities in fundus } \\
\text { photographs }\end{array}$ \\
\hline & reliability $\mathrm{VF}$ test low ${ }^{\dagger}$ & \\
\hline & macular abnormalities in fundus photograph & \\
\hline
\end{tabular}

Briefly, a halogen lamp (12 V, $30 \mathrm{~W}$, Wotan 64260, Osram, Munich, Germany) illuminated a $1.8^{\circ}$ spot on the fovea. Any refractive errors were compensated by adjusting a Badal type front lens system. The light was spectrally filtered (6 mm BG26 Schott AG, Mainz, Germany, $1 \mathrm{~mm}$ Schott UG3, Unaxis TL60, Linos Göttingen, Germany) for the comfort and safety of the subject, and to prevent overloading of the CCD camera (KX85 Apogee Instruments, Inc., Auburn, CA, USA) that served as the detector for the reflected light. The spot intensity was $6.42 \log \mathrm{Td}$; previous calculations showed a maximum safe viewing time of 15 minutes [16]. The filament of the halogen lamp was imaged in the pupil plane of the eye defining a $2.6 \times 1.3 \mathrm{~mm}$ entrance pupil. With a separation of $0.7 \mathrm{~mm}$ below this entrance pupil, a slit-shaped exit pupil of $15 \times 1 \mathrm{~mm}$ formed the input for a prism based imaging spectrometer. The 2-dimensional image projected on the calibrated CCD camera had one directional dimension, resulting from the intensity distribution over the slit-shaped exit pupil. In the other dimension, it contained spectral reflection information. Only light from the central $1.5^{\circ}$ foveal spot was used for analysis. Video observation of pupil plane and retinal plane facilitated proper alignment. A chin rest and temple pads were used to maintain a stable head position. In every included eye, five measurements were performed at the optimal pupil position, i.e. the optical Stiles-Crawford peak

Table 2 Patient characteristics

\footnotetext{
${ }^{\mathrm{a}}$ The maximum known IOP values are probably lower than real IOP values in the past, due to the use of IOP-lowering drugs and/or surgical techniques

${ }^{\mathrm{b}}$ LTP: laser trabeculoplasty, TE: trabeculectomy
}

\begin{tabular}{lllll}
\hline ID & Type of glaucoma & Duration (years) & Max IOP $^{\mathrm{a}}(\mathrm{mmHg})$ & Surgical intervention ${ }^{\mathrm{b}}$ (years ago) \\
\hline 1 & POAG with high IOP & 2 & 34 & LTP (1) \\
2 & POAG with high IOP & 15 & 25 & TE (1) \\
3 & POAG with high IOP & 16 & 32 & LTP (11) \\
4 & POAG with high IOP & 18 & 60 & TE (17) \\
5 & POAG with high IOP & 45 & 18 & TE (11) \\
6 & POAG with high IOP & 40 & 24 & LTP (30) \\
7 & POAG with high IOP & 3 & 26 & none \\
8 & POAG with high IOP & 16 & 26 & none \\
9 & POAG with high IOP & 14 & 28 & none \\
10 & POAG with high IOP & 16 & 33 & none \\
11 & POAG with high IOP & 8 & 35 & TE (4) \\
12 & Normal tension & 2 & 22 & none \\
13 & POAG with high IOP & 26 & 30 & LTP (10) \\
14 & POAG with high IOP & 3 & 48 & none \\
15 & POAG with high IOP & 26 & 41 & LTP (17), TE (9) \\
16 & POAG with high IOP & 10 & 21 & LTP(10), TE (3) \\
17 & Normal tension & 15 & 15 & none \\
18 & Normal tension & 6 & 12 & LTP (6), TE (5) \\
19 & POAG with high IOP & 10 & 40 & TE (1) \\
\hline
\end{tabular}


(a more extensive description of the measurement routine was recently given by Van de Kraats and Van Norren [15]).

The spectra were analyzed with an updated version [15] of the original [17] Van de Kraats fundus reflectance model. The model describes the spectral aspects of light reflected from the fundus for all positions in the pupil (corresponding to various incident angles at the retina), using a limited number of absorbing and reflecting layers. In short, the incoming light is thought to be reflected from four layers: the inner limiting membrane (RILM), the discs in the outer segments of the cone photoreceptors (Rd), the pigment epithelium, and the choroid. In the model, the light traveling through the eye is absorbed by four layers in the eye with known spectral characteristics: media, macular pigment (DMP), melanin (DMEL) and a blood layer. The light conditions were such that all photopigments were bleached. In the optical model, the densities of these absorbers and the reflectances at the interfaces are optimized to fit the measured spectral reflectance. The recent version of the model simultaneously fits the directional reflection from the cone receptors (optical Stiles-Crawford effect, SCE) and the non-directional reflection from more anterior and posterior layers in a wavelength range of 400 $950 \mathrm{~nm}$. In order to make the model more robust, we fixed two non or minimally age-dependent parameters based on data of 102 healthy subjects [15], as described recently in another study from our laboratory [18]. This applied to $\rho$, a measure for the steepness of the optical SCE in the pupil plane (fixed at 0.149) and dRL, a measure for the amount of Rayleigh scatter losses (fixed at 0.53). The latter parameter was derived from the optical model for the age of 52 years, being the mean age of all study participants. Final eyespecific parameters were obtained by averaging the model outcomes for that eye, resulting from the five measurements.

\section{Statistical analysis}

Given the relatively low number of subjects in both the control group and POAG group, a Shapiro-Wilk test was used to test whether our study parameters showed a normal distribution. Because several parameters, including main study parameter $\mathrm{Rd}$, deviated from a normal distribution, the non-parametric Kolmogorov-Smirnov Z-test was used to detect any differences between POAG patients and agematched control subjects with regard to general, optical model, and OCT parameters. The same test was also used to test any differences in Rd and RILM between POAG patients with and without any past surgical intervention. In the POAG patients, Spearman's correlation coefficient rho was computed to verify any linear relationship between Rd and the sensitivity of the central point on 8-degree VF examination. In both participant groups, the same test was used to check whether a linear relationship existed between
Rd and LogMAR BCVA. We finally used Spearman's rho to test whether Rd showed a linear correspondence with RILM in both research groups.

All statistical analyses were performed by means of SPSS for Windows, release 15.0 (SPSS Inc., Chicago, IL, USA).

\section{Results}

Typical examples of single measurements in a healthy 59-year-old eye, and in a POAG eye of the same age are presented in Fig. 1a. The optical SCE shows a Gaussianshaped light output across the pupil. The amplitude of the optical SCE is much smaller in the POAG eye than in the healthy control eye. The spectral reflectances of the same eyes are presented in Fig. 1b. At short wavelengths, the reflectance is very low because of absorption by the lens and the macular pigment. At longer wavelengths $(>550 \mathrm{~nm})$, the reflectance increases by decreasing absorption of melanin and blood; finally $(>850 \mathrm{~nm})$, the reflectance decreases by absorption of water.

At wavelengths below $600 \mathrm{~nm}$ blood absorption is high, whereas above 600 blood absorption is low. Due to these spectral absorption characteristics, only light with wavelengths above $600 \mathrm{~nm}$ will reflect from the choroid. Since this light has no direction, there will be less difference between patients and controls for wavelengths above $600 \mathrm{~nm}$. For wavelengths below $600 \mathrm{~nm}$, hardly any light is reflected from the choroid. Here, the main contribution to the light that is reflected out of the eye is from the photoreceptors. Thus, differences between patients and controls will be more pronounced for wavelengths below $600 \mathrm{~nm}$, as is clearly visible in Fig 1b. Of course, there may be additional differences such as the macular pigment optical density or lens density. However, they exist only in individual cases, since they do not differ between the group of patients and the controls (see further).

Note that the reflectance curves represent the cone photoreceptor reflectance as measured outside the eye, influenced by absorbing media. In contrast, $\mathrm{Rd}$ (and $\mathrm{R}_{\mathrm{ILM}}$, not shown) is a model outcome corrected for losses in the eye media, including the eye lens. Thus, $\mathrm{Rd}$ values (presented in Table 2, not shown in Fig. 1) can not be directly compared to the percentage reflectance shown in Fig. 1.

Table 3 presents the comparisons between general parameters, model outcomes and OCT outcomes for the POAG patients and the control subjects. The optical model also provided information on the goodness-of-fit of the overall model estimation to the measurement data by means of a chi-square value.

Of all model outcomes, only Rd and RILM were significantly lower in the POAG patients than in the control 


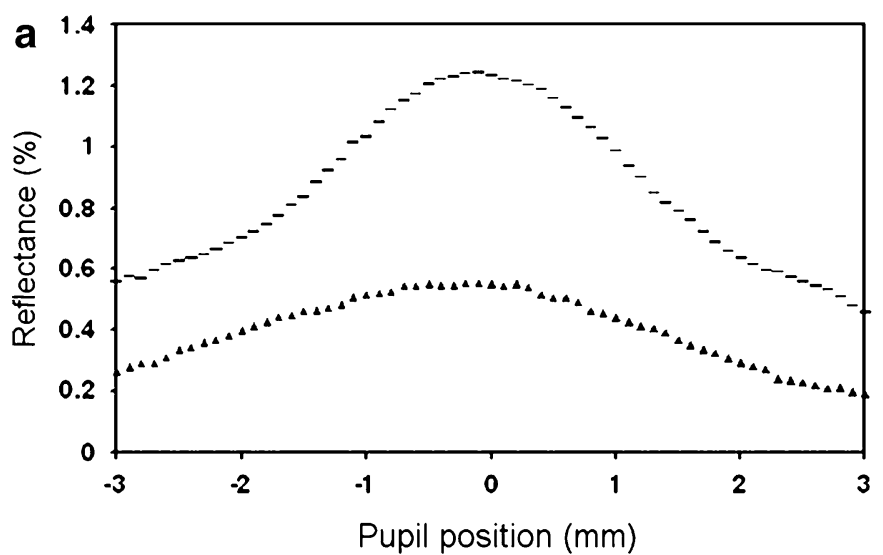

Fig. 1 Directional and spectral reflectance curves of a healthy subject and a POAG patient. a Pupil profiles at $540 \mathrm{~nm}$ of a 59 -year-old healthy man (dashes) and a male POAG patient of the same age (triangles). The pupil profiles show a Gaussian-shaped directional reflection originating from the foveal cone photoreceptors $(\sim$ optical $\mathrm{SCE}$ ), on a diffuse non-directional background. Although present, this directional component is much smaller in the POAG patient. b Spectral reflectance curves of the same subjects at the pupil position

subjects. No difference in chi-square value was seen between the control and the POAG eyes.

In Fig. 2, boxplots of Rd and RILM in both control and POAG eyes are presented.

In four POAG patients, only a laser trabeculoplasty had been performed, in five POAG patients only a trabeculectomy had been performed, and three POAG patients had undergone both interventions in the past. The remaining seven POAG patients had only been treated conservatively. Median Rd (range) was $1.24(0.38-1.88) \%$ in the POAG patients who had undergone a trabeculectomy (with or without history of

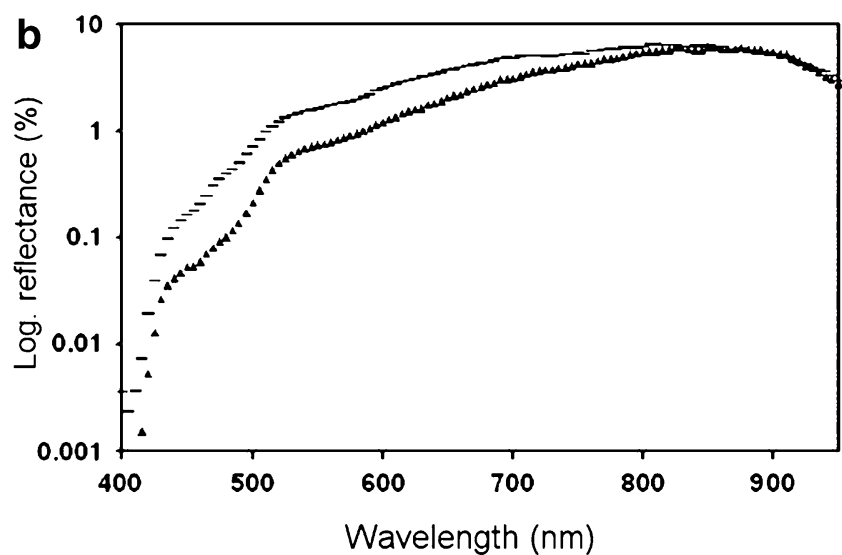

where the optical SCE is at its maximum. At the short wavelengths, the reflection is very low because of the absorption in the eye lens. Around $460 \mathrm{~nm}$ the macular pigment reduces reflectance. At longer wavelengths, the reflectance is seen to increase because of decreasing absorption by melanin and blood. At yet longer wavelengths, water absorption reduces the reflectance. Note that the reflectance around $540 \mathrm{~nm}$ (Fig. 1b) is lower in the POAG patient than in the control subject, in accordance with Fig. 1a (for details see the text)

laser trabeculoplasty) in the past. Median Rd (range) was 1.17 $(0.08-3.60) \%$ in those only treated conservatively. There was no difference in $R_{d}$ between patients who had undergone a laser trabeculoplasty and/or a trabeculectomy in the past and those only treated conservatively $(P=0.60)$. Also, there was no difference in Rd between patients who had undergone a trabeculectomy in the past and those who had not undergone this intervention $(P=0.97)$. Finally, also RILM showed no difference between the latter two patient groups $(P=0.42)$. Note that no postoperative macular edema was documented in the operated patients.

Table 3 Comparison of general, optical model and OCT-related parameters between healthy control subjects and POAG-patients

\begin{tabular}{|c|c|c|c|c|}
\hline & \multirow[t]{2}{*}{ Parameter } & \multicolumn{2}{|l|}{ Value } & \multirow[t]{2}{*}{$P$} \\
\hline & & Control subjects:median (range) & POAG patients:median (range) & \\
\hline \multirow[t]{4}{*}{ General } & $N$ & 34 & 19 & \\
\hline & Age (years) & $55.14(24.72-73.27)$ & $60.05(20.71-77.01)$ & 0.24 \\
\hline & BCVA (LogMAR) & $-0.07(-0.26-0.06)$ & $0.06(-0.12-0.38)$ & 0.001 \\
\hline & IOP $(\mathrm{mmHg})$ & $14(9-21)$ & $15(10-34)$ & 0.86 \\
\hline \multirow[t]{4}{*}{ Optical model } & $N$ & 34 & 19 & \\
\hline & RILM (\%) & $0.15(0.00-1.08)$ & $0.08(0.01-0.29)$ & $<0.001$ \\
\hline & $\mathrm{Rd}(\%)$ & $2.21(0.64-4.93)$ & $1.19(0.08-3.60)$ & 0.003 \\
\hline & chi-square value & $9.31(6.54-28.62)$ & $9.11(4.28-35.79)$ & 0.82 \\
\hline \multirow[t]{5}{*}{$\mathrm{OCT}$} & $N$ & 34 & 16 & \\
\hline & FRT $(\mu \mathrm{m})$ & $169(131-238)$ & $184(131-243)$ & 0.32 \\
\hline & Mean center area thickness $(\mu \mathrm{m})$ & $209(159-270)$ & $208(154-262)$ & 0.67 \\
\hline & Mean inner ring thickness $(\mu \mathrm{m})$ & $275(233-299)$ & $244(177-274)$ & $<0.001$ \\
\hline & Mean outer ring thickness $(\mu \mathrm{m})$ & $233(208-265)$ & $209(162-230)$ & $<0.001$ \\
\hline
\end{tabular}



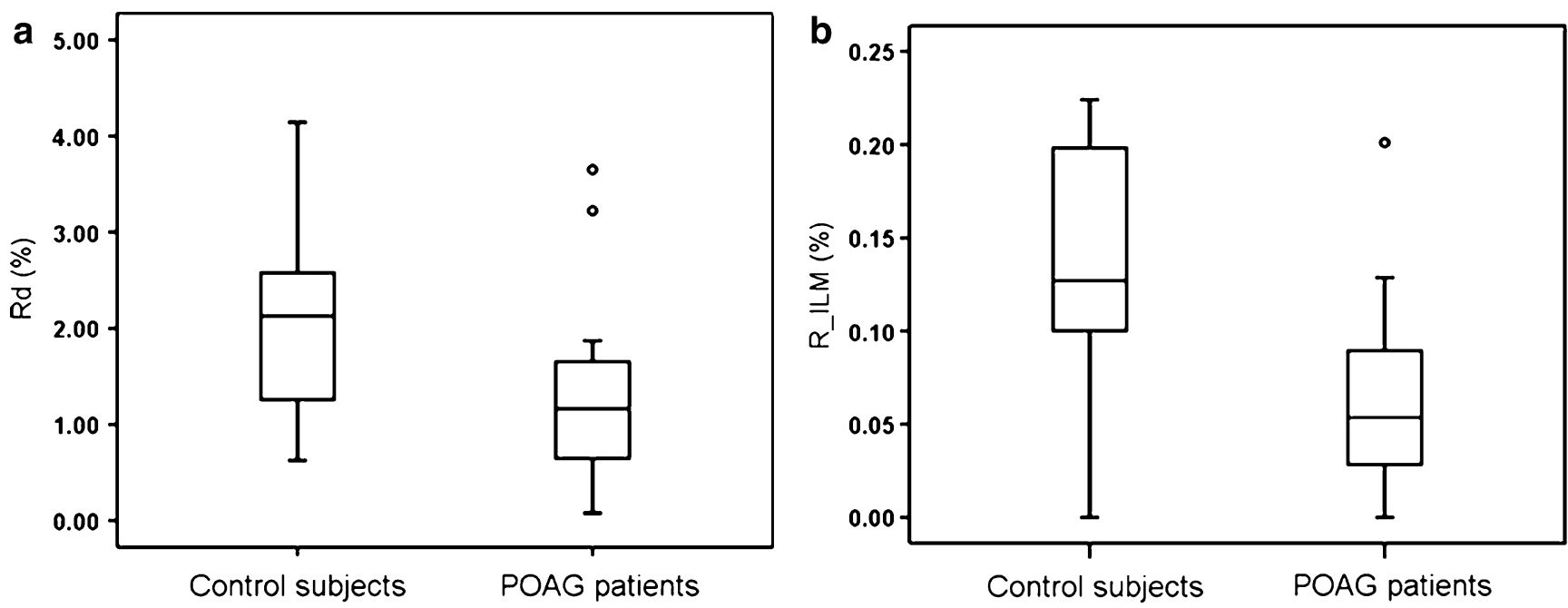

Fig. 2 a Boxplots of Rd in both control subjects and POAG patients. Outliers are shown as small circles. b Boxplots of RILM in control subjects and POAG patients

To find out whether a decrease in $\mathrm{Rd}$ could also be monitored using VF testing, we checked whether a linear relationship existed between $\mathrm{Rd}$ in the POAG patients (foveal sampling field diameter $=1.5^{\circ}$ ) and the sensitivity of the central point (diameter $\sim 2^{\circ}$ ) upon VF testing of these patients, by means of Spearman's nonparametric correlation coefficient: $r h o=0.07 ; P=0.78$.

Using the same nonparametric correlation technique, we also found that $\mathrm{Rd}$ showed no linear association with LogMAR BCVA in the control subjects ( $r h o=-0.27 ; P=$ 0.12 ) nor in the POAG patients ( $r h o=-0.02 ; P=0.95$ ).

Spearman's rho was finally used to test for a possible linear association between Rd and RILM in both research groups. However, no linear association between both model parameters was found in the control subjects $(r h o=-0.07$; $P=0.69)$, or in the POAG patients (rho $=-0.03 ; P=0.89$ ).

\section{Discussion}

We have found that the foveal cone photoreceptors in POAG patients with central visual field defects show a lowered directional reflectance compared to healthy control subjects. Because several studies [15, 17, 19] suggest that the cone outer segments (OS), and/or the connection between outer and inner segments (IS), play a major contribution to the optical SCE, the lowered directional cone-reflectance found in POAG is probably due to either loss of complete foveal cone photoreceptors, as a result of pathologic changes inside the outer segments of these photoreceptors (e.g. loss of individually reflecting outer segment discs), or due to a combination of both processes. However, in several histological studies similar numbers of photoreceptors were counted in glaucoma, as in normal eyes [3-5]. Another indication that the photoreceptor count was not changed in glaucoma was that mean foveal thickness and mean central retinal area thickness were normal in the OCT scans. Any loss of receptors would probably have led to a loss of axons and a thinning of Henle's fiber layer. Some electrophysiological studies [6-10] have also reported outer retinal involvement in glaucoma, further suggesting changes inside cone photoreceptors. We therefore assume that the decrease in $\mathrm{Rd}$ is the result of pathological changes in the structure of the foveal cones.

It is known that choroidal hypoperfusion plays a role in the pathophysiology of POAG. A histological study by Spraul et al. [20] found that post-mortem eyes with advanced glaucomatous damage after long-standing POAG displayed a lower density of the capillaries of the choriocapillaris in the macular choroid as compared to control eyes. Because the outer retina fully depends on the choriocapillaris for its very high oxygen consumption, a lowered foveal choriocapillary density might lead to foveal outer retinal ischemia in advanced POAG, which might in turn affect the foveal cone outer segments.

The only other model parameter that was significantly lower in POAG was RILM, indicating a decreased reflectance of the ILM in POAG at the central $1.5^{\circ}$. As stated before, the central retinal thickness was normal, contrary to the reduced thickness at greater eccentricities (Table 3). Such changes have been observed before [21], and have been explained by losses in cell layers that are not present at the fovea. With an undisturbed underlying Henle fiber layer, it can be thought that the decreased ILM reflectance is caused by structural changes (e.g. wrinkling) of the ILM layer itself. We speculate that loss of ganglion cells further away may cause mechanical stress, even on the 
central ILM. Since we found no linear relationship between $\mathrm{Rd}$ and RILM, these two phenomena are probably independent: Changes in $\mathrm{Rd}$ are related to inner retinal changes, and changes in RILM are related to changing superficial layers away from the fovea.

Best-corrected visual acuity was decreased in the POAG patients. The decrease in Rd in the POAG patients probably bears no relation to the lowered BCVA. A linear relationship between Rd and the BCVA was neither found in the healthy control subjects, nor in the POAG patients. A more likely explanation is changes in more central parts of the visual system than the photoreceptors, in particular the ganglion cell layer.

Other factors that might explain the lowering of Rd and RILM in our POAG group are that an unstable fixation due to poor visual acuity, or an eccentric fixation due to a central scotoma might lead to a lower value of $\mathrm{Rd}$. However, the lowest BCVA in our POAG group was 0.38 LogMAR (Snellen: 42/100), good enough for a stable fixation of the bright target. Because in the POAG group only five out of 19 patients had a significantly decreased sensitivity of the central point in their VF examination, a decreased $\mathrm{Rd}$ due to eccentric fixation is also unlikely. In addition, we found no linear relation between $R_{d}$ and the sensitivity of the central point on VF in the POAG patients. The scenario that surgical interventions have been responsible for a lower Rd and RILM in glaucoma is also unlikely, because we found no difference in Rd and RILM between POAG patients who were treated conservatively and those treated surgically. Finally, it can be thought that the lens density is higher in POAG patients who have undergone a surgical intervention in the past, thus influencing the model outcomes Rd and RILM in the total POAG group. As stated before, $\mathrm{R}_{\mathrm{d}}$ and $\mathrm{R}_{\mathrm{ILM}}$ are model outcomes corrected for losses in eye media. In addition, DLO, density of the aging lens component in the model, did not differ significantly between control subjects and POAG patients (median DLO [range] in control subjects was $0.53[0.21-1.68]$ vs 0.60 $[0.12-1.76]$ in POAG patients, $P=0.47$ ). Severe nuclear sclerosis or cataract would also have led to reflectance data deviating, in the pupil plane, from smooth Gaussian profiles (cf. Fig. 1), and thus to bad model fits. However, we found no difference in goodness of fit (chi-square) between control subjects and POAG patients.

In conclusion, we found a reduced optical StilesCrawford effect in POAG patients with severe central visual field defects. Primary open-angle glaucoma therefore affects the integrity of the cone outer segments. Although not proven by this study, decreased choroidal blood flow might underlie these changes. In addition to changes in foveal cone photoreceptors, a lowered reflectance at the central ILM layer was found in POAG, possibly related to structural changes of this layer in POAG. Optical coherence tomog- raphy showed no thinning of the fovea in POAG, but retinal thinning was present at the inner and outer parafoveal rings.

Acknowledgements The authors wish to thank dr. G.J. van Meel for his help with the inclusion of glaucoma patients.

Open Access This article is distributed under the terms of the Creative Commons Attribution Noncommercial License which permits any noncommercial use, distribution, and reproduction in any medium, provided the original author(s) and source are credited.

\section{References}

1. Quigley HA (1996) Number of people with glaucoma worldwide. Br J Ophthalmol 80:389-393

2. Panda S, Jonas JB (1992) Decreased photoreceptor count in human eyes with secondary angle-closure glaucoma. Invest Ophthalmol Vis Sci 33:2532-2536

3. Kendell KR, Quigley HA, Kerrigan LA, Pease ME, Quigley EN (1995) Primary open-angle glaucoma is not associated with photoreceptor loss. Invest Ophthalmol Vis Sci 36:200-205

4. Wygnanski T, Desatnik H, Quigley HA, Glovinsky Y (1995) Comparison of ganglion-cell loss and cone loss in experimental glaucoma. Am J Ophthalmol 120:184-189

5. Nork TM, Verhoeve JN, Poulsen GL, Nickells RW, Davis MD, Weber AJ, Vaegan SSH, Lemley HL, Millecchia LL (2000) Swelling and loss of photoreceptors in chronic human and experimental glaucomas. Arch Ophthalmol 118:235-245

6. Alvis DL (1966) Electroretinographic changes in controlled chronic open-angle glaucoma. Am J Ophthalmol 61:121-131

7. Fazio DT, Heckenlively JR, Martin DA, Christensen RE (1986) The electroretinogram in advanced open-angle glaucoma. Doc Ophthalmol 63:45-54

8. Holopigian K, Seiple W, Mayron C, Koty R, Lorenzo M (1990) Electrophysiological and psychophysical flicker sensitivity in patients with primary open-angle glaucoma and ocular hypertension. Invest Ophthalmol Vis Sci 31:1863-1868

9. Odom JV, Feghali JG, Jin JC, Weinstein GW (1990) Visual function deficits in glaucoma-electroretinogram pattern and luminance nonlinearities. Arch Ophthalmol 108:222-227

10. Vaegan GSL, Goldberg I, Buckland L, Hollows FC (1995) Flash and pattern electroretinogram changes with optic atrophy and glaucoma. Exp Eye Res 60:697-706

11. Frishman LJ, Shen FF, Du L, Robson JG, Harwerth RS, Smith EL 3rd, Carter-Dawson L, Crawford ML (1996) The scotopic electroretinogram of macaque after retinal ganglion cell loss from experimental glaucoma. Invest Ophthalmol Vis Sci 37:125141

12. Colotto A, Falsini B, Salgarello T, Iarossi G, Galan ME, Scullica L (2000) Photopic negative response of the human ERG: Losses associated with glaucomatous damage. Invest Ophthalmol Vis Sci 41:2205-2211

13. Holopigian K, Greenstein VC, Seiple WM, Hood DC, Ritch R (2000) Electrophysiologic assessment of photoreceptor function in patients with primary open-angle glaucoma. J Glaucoma 9:163168

14. Zagers NPA, Berendschot TTJM, van de Kraats J, van Norren D (2001) A new type of reflectometer: Foveal reflection analyzer. Invest Ophthalmol Vis Sci 42:S702-S702

15. van de Kraats J, van Norren D (2008) Directional and nondirectional spectral reflection from the human fovea. J Biomed Opt 13:024010 
16. Commission on Optical Radiation (1993) Health based exposure limits for electromagnetic radiation in the wavelength region from 100 nanometer to 1 millimeter. Health Council of the Netherlands, The Hague, Report 1993/09:98

17. van de Kraats J, Berendschot TTJM, van Norren D (1996) The pathways of light measured in fundus reflectometry. Vision Res 36:2229-2247

18. Kanis MJ, Wisse RPL, Berendschot TTJM, van de Kraats J, van Norren D (2008) Foveal cone-photoreceptor integrity in aging macula disorder. Invest Ophthalmol Vis Sci 49:2077-2081

19. Gao WH, Cense B, Zhang Y, Jonnal RS, Miller DT (2008) Measuring retinal contributions to the optical Stiles-Crawford effect with optical coherence tomography. Optics Express 16:6486-6501

20. Spraul CW, Lang GE, Lang GK, Grossniklaus HE (2002) Morphometric changes of the choriocapillaris and the choroidal vasculature in eyes with advanced glaucomatous changes. Vision Res 42:923-932

21. Guedes V, Schuman JS, Hertzmark E, Wollstein G, Correnti A, Mancini R, Lederer D, Voskanian S, Velazquez L, Pakter HM, Pedut-Kloizman T, Fujimoto JG, Mattox C (2003) Optical coherence tomography measurement of macular and nerve fiber layer thickness in normal and glaucomatous human eyes. Ophthalmology 110:177-189 